\title{
ATRATIVIDADE DO MERCADO MADEIREIRO AO INVESTIMENTO PRIVADO NO ESTADO DO PARÁ
}

\author{
Silmara Ribeiro Leal ${ }^{1}$, Douglas Valente de Oliveira ${ }^{2}$, Laura Fernanda de Lima Lobato ${ }^{3}$, Juliana Mendes de \\ Oliveira ${ }^{4}$ e Rommel Noce $^{5}$.

\footnotetext{
${ }^{1}$ Acadêmica do curso de Engenharia Florestal, Instituto de Biodiversidade e Florestas (IBEF), Universidade Federal do Oeste do Para (UFOPA), Santarém, Pará, Brasil, mribeiroleal@hotmail.com

${ }^{2}$ Acadêmico do curso de Engenharia Florestal, Instituto de Biodiversidade e Florestas (IBEF), Universidade Federal do Oeste do Para (UFOPA), Santarém, Pará, Brasil, douglasvalentedeoliveira@hotmail.com

${ }^{3}$ Acadêmica do curso de Engenharia Florestal, Instituto de Biodiversidade e Florestas (IBEF), Universidade Federal do Oeste do Para (UFOPA), Santarém, Pará, Brasil, laura.fl@ @hotmail.com

${ }^{4}$ Doutora em Ciências e Tecnologia da Madeira, Instituto de Biodiversidade e Florestas (IBEF), Universidade Federal do Oeste do Para (UFOPA), Santarém, Pará, Brasil, julianameoli@yahoo.com.br

${ }^{5}$ Doutor em Ciência Florestal, Instituto de Biodiversidade e Florestas (IBEF), Universidade Federal do Oeste do Para (UFOPA), Santarém, Pará, Brasil, noce.rommel@gmail.com
}

RESUMO: Objetivou-se analisar a relação risco-retorno dos principais municípios produtores de madeira em tora no período de 2008 a 2014. Admitiu-se a taxa geométrica de crescimento (TGC) como indicativo de retorno e o coeficiente de variação (CV) como indicativo de risco. A atratividade do mercado madeireiro ao investimento privado no estado do Pará mostrou-se bastante variado entre os municípios avaliados. A relação risco/retorno coerente possui valores positivos para a expectativa de retorno, ou seja, atrai investimento, entretanto, nos municípios que apresentaram valores negativos, à relação risco/retorno revelou-se incoerente e consequentemente não atrairia o investimento privado. Observou-se ainda um mercado atrativo a diferentes perfis de investidores, classificados como avesso ao risco, tradicional e propenso ao risco. A maioria dos municípios é classificada como atrativa ao investidor tradicional. De acordo com os resultados, conclui-se que o mercado madeireiro dos municípios do estado do Pará em sua grande maioria é coerente e mais atrativo ao investimento privado de perfil tradicional.

PALAVRAS-CHAVE: Economia florestal, Mercado madeireiro, Risco e retorno.

\section{ATTRACTIVENESS OF THE TIMBER MARKET TO PRIVATE INVESTMENT IN THE STATE OF PARÁ}

\begin{abstract}
The objective of this study was analyze the risk-return of the main timber producing municipalities from 2008 to 2014. The geometric growth rate (TGC) was used as an indicator of return and the coefficient of variation (CV) as indicative of risk. The attractiveness of the timber market to private investment in the state of Pará was quite varied among the municipalities evaluated. The coherent risk/return ratio has positive values for the expectation of return, that is, it attracts investment, however, in the municipalities that presented negative values, the risk/return relationship was incoherent and consequently would not attract private investment. There was also an attractive market for different
\end{abstract}


investor profiles classified as risk averse, traditional and risk prone. Most municipalities are classified as attractive to the traditional investor. According to the results, it is concluded that the timber market of the municipalities of the state of Pará is in large part coherent and more attractive to private investment of a traditional profile.

KEYWORDS: Forest economy, Risk and return, Timber market.

\section{ATRACCIÓN DEL MERCADO MADERERO A LA INVERSIÓN PRIVADA EN EL ESTADO DE PARÁ}

RESUMEN: Se objetivo analizar la relación riesgo-retorno de los principales municipios productores de madera en tora en el período de 2008 a 2014. Se admitió la tasa geométrica de crecimiento (TGC) como indicativo de retorno y el coeficiente de variación (CV) indicativo de riesgo. El atractivo del mercado maderero a la inversión privada en el estado de Pará se mostró bastante variado entre los municipios evaluados. La relación riesgo / retorno coherente tiene valores positivos para la expectativa de retorno, es decir, atrae inversión, sin embargo, en los municipios que presentaron valores negativos, a la relación riesgo / retorno se reveló incoherente y consecuentemente no atraería la inversión privada. Se observó un mercado atractivo a diferentes perfiles de inversores, clasificados como revés al riesgo, tradicional y propenso al riesgo. La mayoría de los municipios se clasifican como atractivos al inversor tradicional. De acuerdo con los resultados, se concluye que el mercado maderero de los municipios del estado de Pará en su gran mayoría es coherente y más atractivo a la inversión privada de perfil tradicional.

PALABRAS CLAVE: Economía forestal, Mercado maderero, Riesgo y retorno.

O setor florestal no Brasil participa significativamente na economia brasileira. Fornece produtos para consumo direto ou exportação, gerando impostos e empregos para a população além de atuar na conservação e preservação dos recursos naturais (ABIMCl, 2009).

O Pará é o principal Estado madeireiro do país, destacando-se tanto no consumo de madeira como na geração de empregos. Sendo que a produção contribui para o superávit nacional, além disso, possui áreas destinadas às concessões estadual e federal com grande estoque de recursos naturais (PEREIRA et al., 2011; SANTANA et al., 2011b; SANTANA et al., 2010). Com a efetividade do marco regulatório da exploração florestal na Amazônia, que desde o ano 2004 reprimiu intensamente o 
desmatamento e a extração ilegal de madeira em tora, somado aos efeitos da crise econômica internacional a partir do ano 2007 e com a diminuição no mercado paraense de produtos madeireiros, houve grande impacto na indústria madeireira e comprometimento do abastecimento do mercado (SANTANA et al., 2011a).

Para resolver o problema de abastecimento e recuperar o setor florestal após a crise, o Instituto de Desenvolvimento Florestal do Estado do Pará (IDEFLOR) implementou a política de gestão de florestas públicas, por meio de contratos de concessão florestal, indicada para a viabilização da extração manejada de madeira em tora pela iniciativa privada. Neste cenário, os contratos de concessão passaram a atuar como o principal mecanismo de regulação da exploração florestal e de promoção do desenvolvimento da cadeia produtiva de madeira em tora no estado do Pará, por oportunizar o fornecimento de matéria prima de origem legal para a indústria madeireira com vantagens comparativa e competitiva sustentáveis. (IDEFLOR, 2009; SANTANA et al., 2011b).
As empresas que possuírem os contratos de concessão podem ter vantagem competitiva em relação às que estão fora do processo e às demais concorrentes no mercado internacional de madeira tropical, uma vez que os preços da madeira em pé refletem no custo de oportunidade da atividade florestal manejada (SANTANA et al., 2012). Porém, segundo Almeida et al. (2010), algumas dificuldades são enfrentadas para efetivar implantação de um manejo florestal sustentável como, por exemplo, necessidade de alto investimento inicial, à burocracia dos órgãos reguladores, à concorrência injusta com madeireiras clandestinas e à falta de segurança fundiária na região amazônica.

Avaliação da dinâmica da atividade florestal pela iniciativa privada mostra que o setor exige altos investimentos nos primeiros anos. Fornecendo, em curto prazo, baixas taxas de retorno inicial devido ao lento amadurecimento da floresta. Dessa maneira, tende a permitir o retorno do capital em longo prazo. Sendo de grande importância analisar a relação risco-retorno dos produtos florestais, no sentido de contribuir para o 
entendimento da atratividade de capital privado para o setor (SILVA, 2007).

O risco pode ser entendido basicamente como a possibilidade de prejuízo financeiro referindo-se a variabilidade do retorno associado a certo ativo. Quanto menor a variabilidade, menor será o risco associado a um determinado ativo (GITMAN, 2010). Segundo o autor, a discussão sobre atratividade ao investimento privado estará fundamentada na coerência da relação risco/retorno nos diferentes mercados, classificados como indicadores da atratividade ao investimento privado. Espera-se que o retorno seja positivo para que a relação risco/retorno seja considerada coerente.

Dessa forma o presente trabalho teve como objetivo analisar a relação riscoretorno dos principais municípios do estado do Pará produtores de madeira em tora no período de 2008 a 2014.

Os preços da madeira em tora dos municípios do estado do Pará, consultados no portal do Instituto Brasileiro de Geografia e Estatística (IBGE), foram estruturados em séries históricas com periodização anual. Após corrigir e filtrar os dados, foram calculados o coeficiente de variação e a taxa de crescimento geométrico para cada município, criando a oportunidade de avaliar graficamente o desenvolvimento do risco e retorno durante os anos observados.

O cálculo das taxas geométricas de crescimento foi executado pelo software GRETL, no qual foi estabelecido, estatisticamente, por regressão linear simples, através da seguinte expressão:

$$
T G C=(\text { Ant } \log \beta-1) \times 100
$$

Sendo:

TCG = Taxa geométrica de crescimento; $\beta=$ Coeficiente de regressão.

Os valores de $\beta$ serão estimados com o ajuste das equações de tendência para cada variável observando o ocorrido em cada município.

Sendo:

$$
\log Y=a+\beta T
$$

Onde:

Y = variável;

$\mathrm{a}$ = constante da regressão; e

$\mathrm{T}=$ tempo

O coeficiente de variação foi alcançado pelo quociente do desvio padrão anual 
dos preços e a média aritmética da serie, de acordo com a fórmula que segue:

$$
C V=\frac{\sigma}{\bar{x}}
$$

Onde:

CV = Coeficiente de variação;

$\sigma=$ desvio padrão;

$\bar{x}=$ média aritmética.

Os resultados foram observados em gráfico de dispersão, onde o risco associado foi admitido como o do coeficiente de variação e o retorno como a taxa de crescimento geométrico. Este procedimento já foi empregado na área florestal, permitindo verificar se a madeira serrada de diferentes espécies tropicais tem relação risco-retorno coerente (NOCE et al., 2010). Utilizou-se Microsoft Excel e software GRETL para a execução de todos os tratamentos de dados.

O comportamento do preço do $\mathrm{m}^{3}$ da madeira em tora no período de tempo analisado nos observados resultados positivos com tendência de crescimento na maioria dos locais.

Para uma atividade captar recursos é fundamental que mostre relação positiva entre suas taxas de risco e retorno (NOCE et al., 2010). A atratividade do mercado madeireiro ao investimento privado no estado do Pará mostrou-se bastante variada entre os municípios, o que pode ser verificado na Figura 1. Vale ressaltar que a maioria dos municípios do estado do Pará se apresenta relação risco/retorno coerente, ou seja, é atrativa ao investimento privado. Enquanto a minoria dos municípios, que apresentaram expectativas de retorno negativas, não seria atrativa ao investimento privado.

Além disso, observa-se um mercado atrativo a diferentes perfis de investidores, sendo classificado como avessos ao risco $(C V<0,11)$, tradicionais $(\geq 11, \leq 46)$ e propensos ao risco (CV $\geq 47)$ (Figura 2).

investidor de perfil avesso ao risco possui baixa tolerância ao risco e admite níveis menores de retorno de seus investimentos, sendo mais sensível a perdas do que ganhos. O tradicional prefere uma relação mais flexível entre risco e retorno. Busca, quando atua no mercado de capitais, uma carteira equilibrada onde parte de seus investimentos estariam assegurados em ativos de baixo ou baixíssimo risco, porém aloca parte do capital admitindo certo nível de risco sob perspectiva de maiores 
ganhos. O propenso ao risco tolera altos riscos associados, buscando altos retornos para seus investimentos, valorizando os ganhos de renda. Admite e, por vezes busca os níveis de risco mais elevados na procura pelas maiores expectativas de ganho. A lógica desse investidor funciona pela máxima de quanto maior o risco maior o retorno (ADAMI, 2010).

Figura 1. Relação risco retorno.

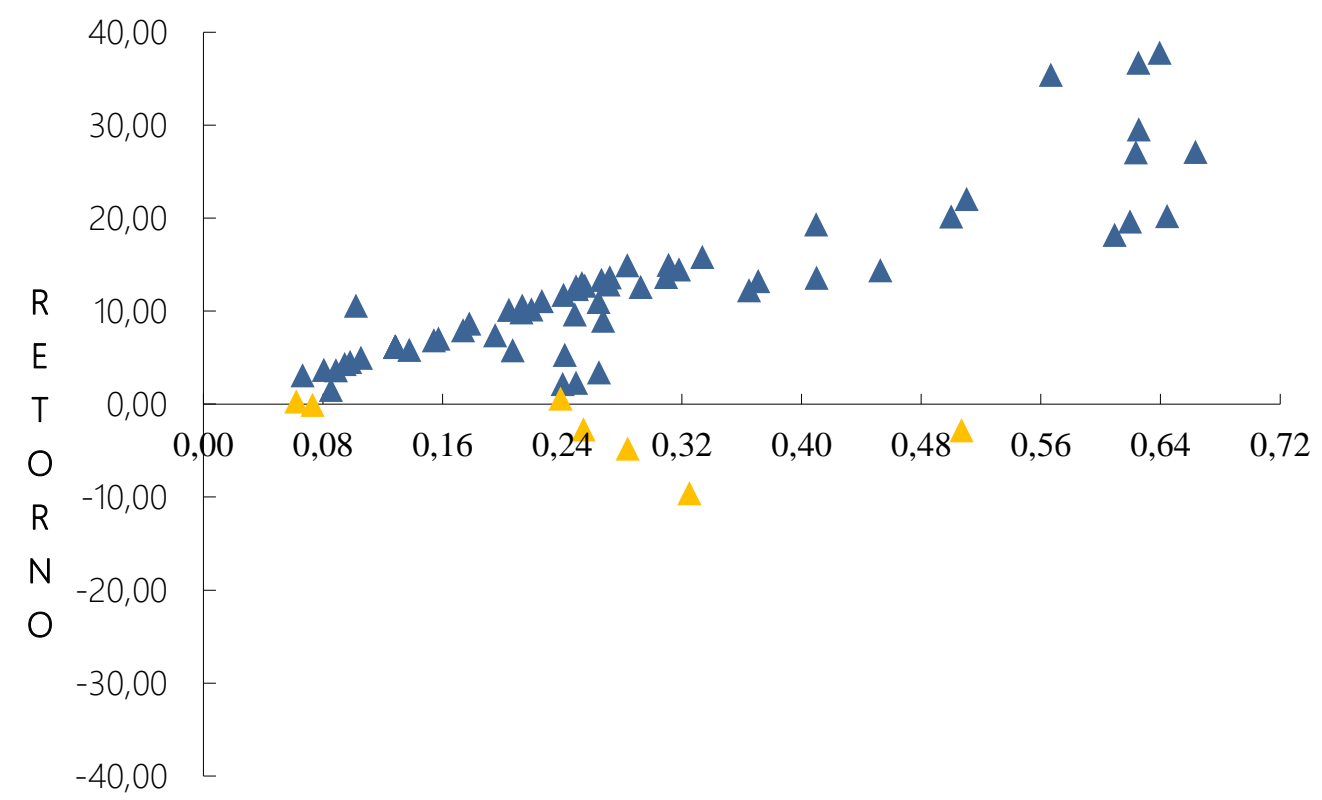

RISCO

$\Delta$ Coerente $\Delta$ Incoerente

Os principais municípios que possuem os mais altos retornos e riscos associados são Abaetetuba (37,82; 0,64), Moju (36,71; 0,63), Barcarena $(35,43 ; 0,57)$ e IgarapéMiri $(29,56 ; 0,63)$, respectivamente. Seriam assim, mais atrativos ao investidor propenso ao risco. Considerando o perfil de investidor avesso ao risco os municípios em destaque são Pau d'Arco
$(4,97 ; 0,11)$, Conceição do Araguaia $(10,58$; $0,10)$, Monte Alegre $(4,57 ; 0,10)$ e Ourilândia do Norte $(4,32 ; 0,09)$. Destacase que o risco estimado é relativamente próximo, dentro do grupo, sendo que o município de Conceição do Araguaia apresenta mais do que o dobro do retorno estimado. 
Figura 2. Perfil do investidor dos principais municípios do Pará.

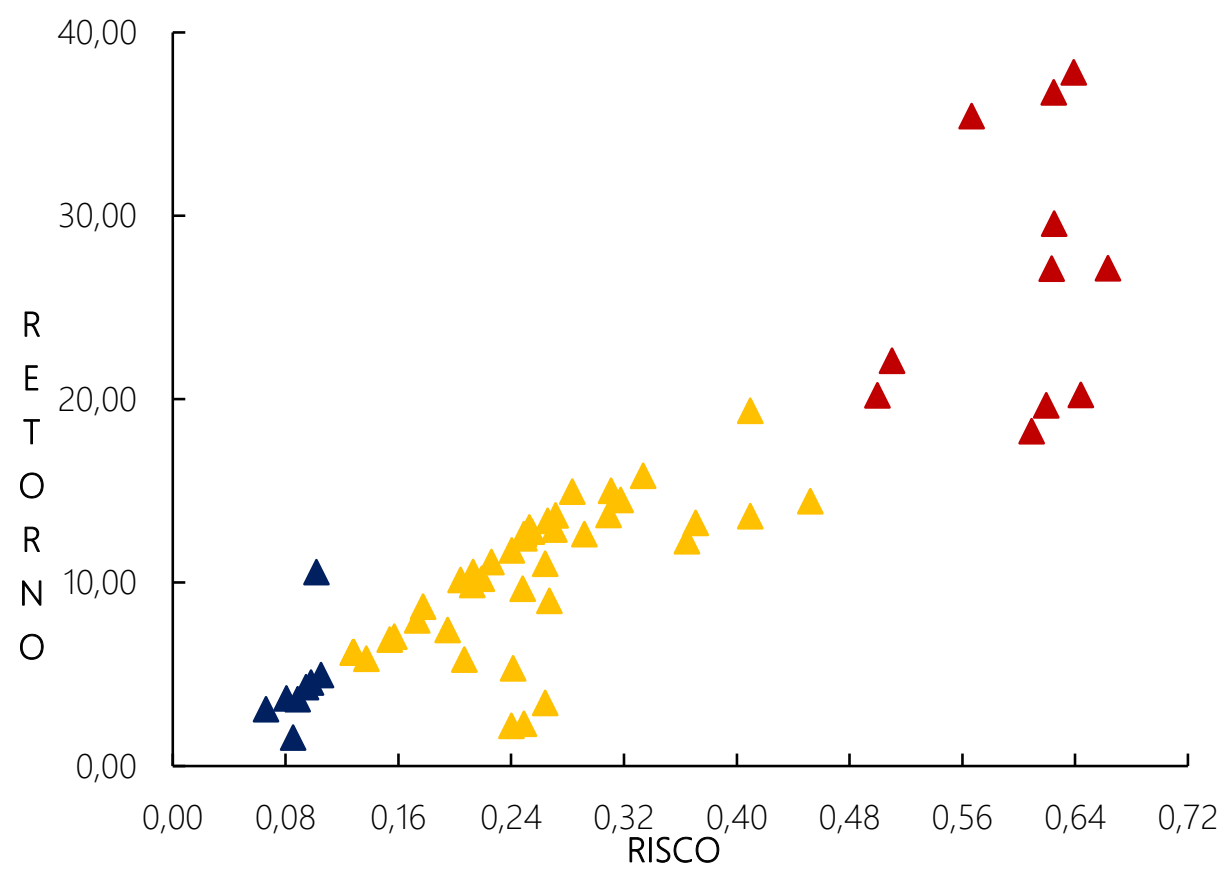

$\Delta$ Avesso ao risco $\Delta$ Tradicional $\Delta$ Propenso ao risco

Tabela 1. Classificação de risco dos municípios do estado do Pará.

\begin{tabular}{|c|c|c|}
\hline Avesso ao risco & Tradicional & Propenso ao risco \\
\hline $\begin{array}{l}\text { Alenquer, Conceição do } \\
\text { Araguaia, Jacareacanga, } \\
\text { Jacundá, Monte Alegre, } \\
\text { Novor Progresso, } \\
\text { Ourilândia do Norte, Pau } \\
\text { d'Arco, Placas e } \\
\text { Redenção }\end{array}$ & $\begin{array}{l}\text { Abel Figueiredo, Acará, Afuá, Água Azul do } \\
\text { Norte, Alenquer, Almeirim, Altamira, Anajás, } \\
\text { Anapu, Aveiro, Bagre, Bannach, Belterra, Bom } \\
\text { Jesus do Tocantins, Brasil Novo, Brejo Grande } \\
\text { do Araguaia, Breves, Bujaru, Cachoeira do Piriá, } \\
\text { Canaã dos Carajás, Chaves, Conceição do } \\
\text { Araguaia, Cumaru do Norte, Curionópolis, } \\
\text { Curralinho, Curuá, Dom Eliseu, Eldorado do } \\
\text { Carajás, Faro, Floresta do Araguaia, Garrafão } \\
\text { do Norte, Goianésia do Pará, Gurupá, Itaituba, } \\
\text { Itupiranga, Jacareacanga, Jacundá, Juriti, } \\
\text { Marabá, Medicilândia, Melgaço, Monte Alegre, } \\
\text { Muaná, Nova Esperança do Piriá, Noval Ipixuna, } \\
\text { Novo Progresso, Novo Repartimento, } \\
\text { Oriximiná, Ourilândia do Norte, Pacajá, } \\
\text { Palestina do Pará, Paragominas, Parauapebas, } \\
\text { Pau d'Arco, Piçarra, Placas, Portel, Porto de } \\
\text { Moz, Redenção, Rondon do Pará e Rurópolis }\end{array}$ & $\begin{array}{l}\text { Baião, Mocajuba, } \\
\text { Cametá, Abaetetuba, } \\
\text { lgarapé-Miri, Moju, } \\
\text { Limoeiro do Aruju, } \\
\text { Prainha, Oeiras do Pará, } \\
\text { Barcarena, Óbidos, Breu } \\
\text { Branco e lpixuna do Pará }\end{array}$ \\
\hline
\end{tabular}

Fonte: Elaborado pelo autor. 
A maioria dos municípios são classificados como tradicionais, ou seja, onde há expectativa de estabilidade e investimento contínuo (Tabela 1). Dentre estes municípios destacam-se em termos de atratividade Curuá (15,82; 0,33), Medicilândia (15,01; 0,31), Melgaço (14,94; 0,28) e Brasil Novo (14,51; 0,32), possuem seus valores de risco próximos e os retornos mais altos.

Operam no Pará 7 empresas madeireiras que possuem o FCS-Forest Stewarship, certificação Florestal (SANTOS; SANTANA, 2009). Algumas delas estão nos municípios de Almerim, Portel, Novo Repartimento e Afuá que apresentaram um retorno razoável, relação risco retorno coerente e atrairiam, a princípio, o investidor tradicional.

A expectativa é de crescimento do setor, visto que na grande maioria dos municípios a atividade madeireira apresenta-se atrativa ao investimento privado. Contemplando inclusive os diferentes perfis de investidores.

Conclui-se que a maioria dos municípios do estado do Pará apresenta uma relação risco/retorno coerente, sendo atrativos ao investimento privado no setor madeireiro. O maior número de municípios, compreendidos na análise, encontram-se atraindo ao investidor de perfil tradicional. Ou seja, atrairiam quem está disposto a assumir certo nível de risco ante a perspectiva de aumentar os seus ganhos.

O investidor de perfil propenso ao risco foi identificado em 13 municípios. Enquanto o de perfil avesso ao risco, que admite sacrificar as oportunidades de rendimentos em favor de baixos riscos de perdas financeiras, foi identificado com a menor frequência em 9 dos 74 municípios analisados.

Denota-se assim, que o perfil do investidor na atividade madeireira no estado do Pará é majoritariamente tradicional.

\section{REFERÊNCIAS}

ASSOCIAÇÃO BRASILEIRA DA INDÚSTRIA DE MADEIRA PROCESSADA MECANICAMENTE - ABIMCI. Estudo setorial 2009 ano base 2008. 2009.

ADAMI, A. C. O. Risco e retorno de investimentos em citros no Brasil. Piracicaba, 2010. 150 p. Tese (Doutorado). Escola Superior de Agricultura "Luiz de Queiroz", Piracicaba, 2010.

ALMEIDA, A. N. de; ANGELO, H.; SILVA, J. C. G. L. da; HOEFLICH, V. A. Mercado de 
madeiras tropicais: substituição na demanda de exportação. Revista Acta Amazonica, v. 40, n. 1, p. 119 - 126, 2010.

BARBOSA, H. F.; REGO, L. J. S.; PIERO, M. E.; NOCE, R.; OLIVEIRA, J. M. de ; GAMA, J. R. V. Risk-return and difference of Ipe wood price in Pará and São Paulo markets. CERNE (UFLA), v. 20, p. 69-72, 2014.

GITMAN, L. J. Princípios de administração financeira. 12. ed. Sao Paulo: Pearson, 2010. 800 p.

IDEFLOR. Instituto de Desenvolvimento Florestal do Estado do Pará. Instrução Normativa No 001/2009. Diário Oficial no 31373. Belém, PA, 4 de março de 2009.

PEREIRA, D.; SANTOS, D.; VERÍSSIMO, A.; SALOMÃO, R. Oferta e demanda de áreas para manejo florestal no Estado do Pará. O estado da Amazônia-Imazon, n. 20, Belém-PA, Ago., 2011.

NOCE, R.; SILVA, M. L.; MENDES, L. M.; SOUZA, A. L.; REZENDE, J. L. P.; CARVALHO, R. M. M. A.; CANTO, J. L.; OLIVEIRA, J. M. Risk-return of native species sawn wood in the state of Pará, Brazil 2003- 2007. Cerne, Lavras, v. 16, n. 2, p. 199-207, 2010.

SANTANA, A. C.; SANTOS, M. A. S.; OLIVEIRA, C. M de. Preço da madeira em pé, valor econômico e mercado de madeira nos contratos de transição do estado do Pará. Belém: IDEFLOR/UFRA, 2010a. 114p.

SANTANA, A. C. de; SANTANA, A. L. de; SANTOS, M. A. S. dos. Influência do desmatamento no mercado de madeira em tora da região Mamuru-Arapiuns, Sudoeste do Pará. Revista de Ciências Agrárias, v.54, n.1, p.44-53, 2011 a.

SANTANA, A. C. de; SANTANA, A. L. de; SANTOS, M. A. S. dos; YARED, J. A. G. Determinação dos preços da madeira em pé para as áreas de florestas públicas da região do baixo amazonas, no estado do Pará. Revista de Estudos Sociais, Cuiabá, v. 13, n. 1, p. 40-51, 2011.

SANTANA, A. C. de; SANTOS, M. A. S. dos; SANTANA, A. L. de; YARED, J. A. G. O valor econômico da extração manejada de madeira no baixo amazonas, estado do Pará. Revista Árvore, Viçosa-MG, v.36, n.3, p.527-536, 2012.

SANTANA, A. C. de; SANTANA, A. L. de.; OLIVEIRA, C. M. de.; SANTOS, M. A. S. DOS.; AMIN, M. M.; COSTA, N. L. O valor econômico da extração de madeira em tora na Ilha do Marajó, Pará. Teoria e Evidência Econômica, v. 22, n. 47, p. 296311, jul./dez. 2016.

SILVA, M. L. da; SILVA, R. F. da. Aplicação da programação dinâmica na substituição de povoamentos florestais. Rev. Árvore, Viçosa, v. 31, n. 6, p. 1063-1072, Dec. 2007. 